\title{
Analysis and Recurrent Calculation of 8th Rank MBF of Maximal Types
}

\author{
Tkachenco V. G. ${ }^{1, *}$, Sinyavsky O. V. ${ }^{2}$ \\ ${ }^{1}$ Institute of Radio, Television, Electronics, Odessa National Academy of Telecommunications, Ukraine \\ ${ }^{2}$ Department of Fundamental Sciences, Odessa Military Academy, Ukraine
}

Received November 7, 2019; Revised December 13, 2019; Accepted December 17, 2019

Copyright $\bigcirc 2020$ by authors, all rights reserved. Authors agree that this article remains permanently open access under the terms of the Creative Commons Attribution License 4.0 International License

\begin{abstract}
This paper is a continuation of the study of monotone Boolean functions (MBFs) of maximal types using MBF partitioning into schemes. When factor out any of the variables out of the brackets, two MBFs are formed: left (in brackets) and right. It proved the possibility of such that take the one of the variables out of the brackets such that any conjunctive clause in the left MBF consists of fewer variables than any conjunctive clause in the right MBF. In addition, the left MBF absorbs the right MBF. For the first time, an important class of MBF of rank 8 - MBF of maximal types was studied and analyzed. The number of MBFs of maximal types of rank 8 and the number of isomorphic classes of such MBFs obtained from pairs of MBFs 7 rank are calculated. An example of the recursive construction MBF 8 rank is shown. Tables and schemes for MBF 8th rank are given. The dependences found between the maximal types MBF $n$th rank and rank $n-1$ make it possible to reduce the enumeration of MBFs by constructing rank 8 equivalence classes from rank 7 equivalence classes. The proposed methods are convenient for the analysis of large MBF ranks.
\end{abstract}

Keywords Monotone Boolean Functions, MBF Types, Maximal Types, MBF Profile, MBF Schemes, MBF Equivalence Classes

\section{Introduction}

In 1897, R. Dedekind published an article [1], in which the number of elements of a distributive lattice with four generators was found. The number $\psi(n)$ of elements of the distributive lattice with $n$ generators coincides with the number of anti-chains in the unit $n$-dimensional cube. In the language of the algebra of logic, $\psi(n)$ is the number of monotone Boolean functions depending on the variables $x_{1}, \ldots, x_{n}$. The problem of computing $\psi(n)$ is usually called the Dedekind problem. As it turned out, this problem is rather difficult and cannot be solved within the framework of the traditional method of generating functions. There was an attempt to computation the Dedekind number $D(n)$ through the number of classes of nonisomorphic MBFs. As in the case of $D(n)$, the closed formula for such classes is not known, and in fact only values $p$ to $n=6$ were calculated (see [2]). They, apparently, were obtained by a direct method of enumerating all monotone Boolean functions of $n$ variables, and then sorting them into equivalence classes.

In 1997, Engel introduced the concept of a profile of a monotone Boolean function [3]. T. Stephen and T. Yusun [4] parted the whole set of MBF into classes using the profiles, to find the number of nonisomorphic MBF. They developed an algorithm on Matlab to compute the number of classes of such MBFs of 7 variables. This method is based on the partation of the MBF into profiles, which are defined in [3]. In 1991, Wiedemann [5] calculated the eighth Dedekind number. However, since then, no new results have been achieved in the study and analysis of rank $8 \mathrm{MBF}$.

Independently, the concept of an MBF type similar to the concept of a profile was introduced in [6]. In [7] classification of MBF on types and enumeration of the MBF maximum types is developed. In [8], a new method for recursively calculating the number Dedekind $D(n)$ was developed on the basis of partitioning into MBF equivalence classes and the algebraic properties of MBF blocks were investigated.

In this paper MBFs of the maximum types of 8 rank are for the first time studied and analysed. The method [9] of recurrent calculation of MBF of the maximum type of 8 rank is applied to calculation of their number and the number of classes of equivalence using $\mathrm{MBF}$ of the maximum type of 7 rank. Classification of MBF of the maximum types of 8 rank on schemes is given. Tables by the number of classes of equivalence and on the power of 
these classes are constructed.

\section{Results}

The Boolean function of $\mathrm{n}$ elements is the mapping $f: B^{n} \rightarrow B$, where $B=\{0,1\}$. A monotone Boolean function (MBF) is a Boolean function under the condition: for any $\alpha, \beta \in B^{n}$ such that $\alpha<\beta$, then $f(\alpha)<f(\beta)$. The lattice of all MBFs of $n$ rank is a free distributive lattice of the $n$ rank plus zero MBF (on all sets it is 0 ) and the unit MBF (on all sets is 1). Under the over / under ratio, we understand the order in this lattice of all MBFs. We will consider the Boolean function in the disjunctive normal form (DNF). MBF can be determined through a DNF. A monotone Boolean function is a Boolean function that does not have a negation operation in the form of a DNF, but only a disjunction and conjunction operation.

A vector $T=\left(a_{0}, a_{1}, \ldots, a_{i}, \ldots, a_{n}\right)$ is an MBF type if the $i$-th component of the vector $a_{i}$ is equal to the number of conjunctive clauses in the form of DNF, which consist of $i$ variables, i.e. have length $i$. Several new characteristics were introduced for the type: the number $n$ is called the rank of a type $T$; the number of non-zero component $v-$ weight of a type $T$; the number $j$ of the first nonzero components on the right - the right border of the $\mathrm{T}$; the number $i$ of the first nonzero components on the left - the left border of the $T$; the sum $m$ of all the components of the $T$ - cardinality type $T$.

The type $\mathrm{T}$ is called maximal if, with increasing any of its components by 1 , the resulting vector will not be a type, i.e. there is no $\mathrm{MBF}$, the type of which would be equal to this vector. Conversely, if we subtract one or more units from any component, the resulting vector will also be an MBF type, since we will remove the clauses of the length of the corresponding component from the existing MBF. By definition, this function will be monotonic. Thus, any type can be obtained by subtracting integers from components of one or more maximum types.

We call type $T_{1}^{-1}=\left(a_{n}, a_{n-1}, \ldots, a_{n-i}, \ldots, a_{1}, a_{0}\right)$ the inverse type for type $T_{1}=\left(a_{0}, a_{1}, \ldots, a_{i}, \ldots, a_{n}\right)$. This means that if the MBF $f$ is of type $T_{1}$, then the type of disjunctive complement MBF $g$ is $T_{1}^{-1}$

EXAMPLE 1. The following are the maximum types of ranks from 0 to 4 .

For rank 0, only type (1) is maximal.

For rank 1, the maximal are 2 types: $(0,1)$ and $(1,0)$.

For rank 2, the maximal is 3 types: $(0,0,1),(0,2,0)$ and $(1,0,0)$.

For rank 3 , the maximal is 5 types: $(0,0,0,1),(0,0,3,0)$, $(0,1,1,0),(0,3,0,0)$, and $(1,0,0,0)$.

For rank 4 , the maximal is 10 types: $(0,0,0,0,1),(0,0,0$, $4,0),(0,0,1,2,0),(0,0,3,1,0),(0,0,6,0,0),(0,1,0,1,0)$, $(0,1,3,0,0),(0,2,1,0,0),(0,4,0,0,0),(1,0,0,0,0)$
We call the types of views $(0,0, \ldots, 0),(1,0, \ldots, 0),(0,0, \ldots, 0,1)$ zero, left and right, respectively. For the zero type, the right and left units coincide and are equal to (1).

Let's call a pair of types $T_{1}$ and $T_{2}$ rank $n$ admissible if the right boundary of $j\left(T_{1}\right)$ is strictly less than the left boundary of $i\left(T_{2}\right)$, i.e. All conjunctive clauses of MBF of type $T_{1}$ are strictly less than any clauses of MBF of type $T_{2}$.

For any admissible pair of types, a shift-sum operation is defined:

$$
\begin{aligned}
& T=T_{1} \circ T_{2}=\left(a_{0}, a_{1}, \ldots, a_{n}\right) \circ\left(b_{0}, b_{1}, \ldots, b_{n}\right)= \\
& =\left(b_{0}, a_{0}+b_{1}, \ldots, a_{n-1}+b_{n}, a_{n}\right)=\left(c_{0}, c_{1}, \ldots, c_{n+1}\right)
\end{aligned}
$$

If $T=T_{1} \circ T_{2}, T^{-1}=T_{2}^{-1} \circ T_{1}^{-1}$

Such an operation with a zero vector $T_{0}=(0,0, \ldots, 0)$ it is possible to carry out both on the right, and at the left:

$$
\begin{aligned}
& T=T_{1} \circ T_{0}=\left(a_{0}, a_{1}, \ldots, a_{n}\right) \circ(0,0, \ldots, 0)=\left(0, a_{0}, \ldots, a_{n-1}, a_{n}\right) \\
& T=T_{0} \circ T_{1}=(0,0, \ldots, 0) \circ\left(a_{0}, a_{1}, \ldots, a_{n}\right)=\left(a_{0}, \ldots, a_{n-1}, a_{n}, 0\right)
\end{aligned}
$$

There are $f_{1}, \ldots, f_{r}$ left MBF $n-1$ rank and $g_{1}, \ldots, g_{m}$ right $\mathrm{MBF} n-1$ rank.

Each MBF $n$th rank $f(n)$ of the maximal type consists of an admissible pair of MBFs $n-1$ rank, left $f(n-1)$ and right $g(n-1)$ connected by separable variable $x_{n}$, $f(n)=f(n-1) x_{n} \vee g(n-1)$.

It is known that conjunctive clause $S$ absorbs conjunctive clause $t$ if all its variables are components of conjunctive clause $t$. We say that MBF $f$ absorbs MBF $g$, if each conjunctive clause $g$ is absorbed by one of conjunctive clause $f$. For the binary representation, it can be written that $f \geq g$, i.e. one function is greater than another. This corresponds to the ratio "greater than or equal" on all elements of a free distributive lattice from MBF of $n$ rank. From this inequality it follows that $f=f \vee g$ and $g=f \wedge g$.

We introduce the concept of an index of separating variables $\lambda$. We construct from two MBFs of $n-1$ rank, which are MBFs of an admissible pair of maximal types MBF $n$ rank $f(n)=f_{n}(n-1) x_{n} \vee g_{n}(n-1)$. A pair of types $T_{1}$ and $T_{2}$ is uniquely determined, because this decomposition is unique [10]. Proved [8] Theorem 1, according to which for any admissible pair of maximal types $T_{1}$ and $T_{2}$ and any $f_{n}(n-1) \in T_{1}$ and $g_{n}(n-1) \in T_{2}$ we have $f_{n}(n-1)>g_{n}(n-1)$, i.e. MBF $f_{n}(n-1)$ absorbs MBF $g_{n}(n-1)$. Suppose that in the same function $f(n)$ we can take other variables out of the brackets except $x_{n}$, i.e. $f(n)=f_{i}(n-1) x_{i} \vee g_{i}(n-1)$. Then $f_{i}(n-$ $1)$ is isomorphic to $f_{n}(n-1), g_{i}(n-1)$ is isomorphic to $g_{n}(n-$ $1), f_{i}(n-1)>g_{i}(n-1), f_{i}(n-1) \in T_{1}, g_{i}(n-1) \in T_{2}$, and the types 
and constitute an admissible pair. We call the number of such variables $x_{i}$ the index of the separating variables $\lambda$.

In [9], it is assumed by default that $\lambda \neq 0$ for all MBFs of maximal types. Further this statement will be proved. For MBFs of arbitrary types, $\lambda=0$ is possible. The simplest such MBF is $h(5)=x_{1} x_{5} \vee x_{2} x_{3} \vee x_{1} x_{2} x_{4} \vee x_{1} x_{3} x_{4}$ of rank 5 of type $(0,0,2,2,0,0)$. When take the $x_{5}$ out of the brackets, we obtain the left $x_{1}$ of type $(0,1,0,0,0)$ and the right side of $x_{2} x_{3} \vee x_{1} x_{2} x_{4} \vee x_{1} x_{3} x_{4}$ of type $(0,0,1,2,0)$. However, the left side does not absorb the right side. In fact, the left side should be taken (before take the $x_{5}$ out of the brackets the identity $x_{2} x_{3}=x_{2} x_{3} \vee x_{2} x_{3} x_{5}$ applies) $x_{1} \vee x_{2} x_{3}$ of type $(0,1,1,0,0)$, and the right side is to be left with $x_{2} x_{3} \vee x_{1} x_{2} x_{4}$ $\vee x_{1} x_{3} x_{4}$ of type $(0,0,1,2,0)$. This pair of types is inadmissible and therefore $\lambda=0$ for MBF $h(5)$. In total, type $(0,0,2,2,0,0,0)$ have 5 equivalence classes with $\lambda=1$ and 2 equivalence classes with $\lambda=0$. In MBF $h(5)$, you can add more conjunctive clauses, i.e. type $(0,0,2,2,0,0)$ is not maximal. However, among MBFs with $\lambda=0$ there are such that any conjunctive clauses that is added to them is absorbed by one of the conjunctive clauses belonging to this MBF. In this way, such MBFs are similar to maximal type MBFs, to which no conjunctions can be added either. We call such MBFs maximal with $\lambda=0$. For example, maximal MBFs with $\lambda=0$ are MBFs $f(5)=x_{1} x_{5} \vee x_{2} x_{3} \vee$ $x_{1} x_{2} x_{4} \vee x_{1} x_{3} x_{4} \vee x_{2} x_{4} x_{5} \vee x_{3} x_{4} x_{5}$ of type $(0,0,2,4,0,0)$ and $g(5)=x_{1} x_{2} \vee x_{1} x_{5} \vee x_{2} x_{5} \vee x_{1} x_{3} x_{4} \vee x_{2} x_{3} x_{4} \vee x_{3} x_{4} x_{5}$ type $(0,0,3,3,0,0)$.

If in some conjunctive clause $A$ of the MBF $f(n)$ of type $T$ there are $i$ variables, then we say that $A$ belongs to a component $i$ of type $T$. Each component $i$ of type $T$ with value $z(i)$ belongs $z(i)$ conjunctive clauses. The variable $x_{\mathrm{i}}$ is separating if, after to take out of the brackets, we obtain the left MBF $f_{1}(n-1)$ of type $T_{1}$ and the MBF $f_{2}(n-1)$ of type $T_{2}$, and $T_{1}$ and $T_{2}$ are the left and right parts of type $T, T_{1}$ and $T_{2}$ - admissible pair of types and $f_{1}(n-1)$ absorbs $f_{2}(n-$ $1)$, i.e. $f_{1}(n-1)>f_{2}(n-1)$. If the variable $x_{\mathrm{k}}$ is not separating, then 2 cases are possible. In the first case, there exists a component $i$ of type $T$ corresponding to component $i-1$ of type $T_{1}$ and containing 2 conjunctive clauses $A$ and $B$, moreover, $x_{k} \in A$ and $x_{k} \notin B$. If the conjunctive clauses $A$ and $B$ do not have common variables, then $\lambda=0$ for $f(n)$. An example of MBF with $\lambda=0$ of the first case is the above MBF of $f(5)$ type $(0,0,2,4,0,0)$.

EXAMPLE 2. We show that the MBF $f(5)=x_{1} x_{5} \vee x_{2} x_{3}$ $\vee x_{1} x_{2} x_{4} \vee x_{1} x_{3} x_{4} \vee x_{2} x_{4} x_{5} \vee x_{3} x_{4} x_{5}$ type $(0,0,2,4,0,0)$ is not a maximal type MBF. To do this, in the conjunctive clause $x_{2} x_{3}$, replace $x_{3}$ with $x_{5}$ and get $f_{1}(5)=x_{1} x_{5} \vee x_{2} x_{5} \vee x_{1} x_{2} x_{4} \vee$ $x_{1} x_{3} x_{4} \vee x_{2} x_{4} x_{5} \vee x_{3} x_{4} x_{5}$. The resulting conjunction $x_{2} x_{5}$ absorbs the conjunction $x_{2} x_{4} x_{5}$, therefore, in $x_{2} x_{4} x_{5}$ we carry out the reverse change, i.e. replace $x_{5}$ with $x_{3}$ and get $f_{2}(5)=$ $x_{1} x_{5} \vee x_{2} x_{5} \vee x_{1} x_{2} x_{4} \vee x_{1} x_{3} x_{4} \vee x_{2} x_{3} x_{4} \vee x_{3} x_{4} x_{5}$. The transition from $f(5)$ to $f_{2}(5)$ is called the first operation. Obviously $f_{1}(5), f_{2}(5)$ and $f(5)$ are of type $(0,0,2,4,0,0)$. But to $f_{2}(5)$, unlike $f(5)$, we can add the conjunction $x_{1} x_{2} x_{3}$. We get MBF $f_{3}(5)=x_{1} x_{5} \vee x_{2} x_{5} \vee x_{1} x_{2} x_{3} \vee x_{1} x_{2} x_{4} \vee x_{1} x_{3} x_{4} \vee$ $x_{2} x_{3} x_{4} \vee x_{3} x_{4} x_{5}$ type $(0,0,2,5,0,0)$. By the definition of the maximal type, neither $f_{2}(5)$ nor $f(5)$ are MBFs of maximal types.

Theorem 1. An $\operatorname{MBF} f(n)$ of type $T$ with $\lambda=0$ of the first case is not an MBF of maximal type.

Proof. By the definition of the first case, there exists a component $i$ of type $T$ corresponding to component $i-1$ of type $T_{1}$ and containing 2 conjunctive clauses $A$ and $B$ that do not have common variables. We apply the first operation to $f(n)$, i.e. in the conjunctive clause $B$ we replace one of the variables with the variable contained in $A$ and obtain the conjunctive clause $B_{1}$. Then, in all conjunctive clauses that the conjunctive clause $B_{1}$ absorbs, we make the reverse change. We will repeat the first operation until the conjunctive clauses $A$ and $B_{i-1}$ have $i-1$ common variables. Conjunctive clauses $A$ and $B$ can absorb $2 C_{n-i}^{j-i} C_{n-2 i}^{j-2 i}$ conjunctive clauses of length $j$. In other words, you can place as much as $C_{n}^{j}-2 C_{n-i}^{j-i}+C_{n-2 i}^{j-2 i}$ conjunctive clauses of length $j$. In the case of conjunctive clauses $A$ and $B_{i-1}$, it is possible to place maximally $C_{n}^{j}-2 C_{n-i}^{j-i}+C_{n-i-1}^{j-i-1}$ conjunctive clauses of length $j$. The last expression, even for $j=i+1$, is greater than the previous one. This means that type $T$ is not maximal. The theorem is proved.

In the second case, with $\lambda=0$, there exists a component $i$ of type $T$ corresponding to component $i-1$ of type $T_{1}$ and containing 3 conjunctive clauses $A, B$ and $C$ that do not have common variables, and any variable $x_{k}$ belonging to two conjunctive clauses from $A, B$ and $C$ belongs to also some conjunctive clause $D$, which contains component $i+$ 1 of type $T$. An example of MBF with $\lambda=0$ of the second case is the above MBF $g(5)$ of type $(0,0,3,3,0,0)$.

EXAMPLE 3. We show that the MBF $g(5)=x_{1} x_{2} \vee x_{1} x_{5}$ $\vee x_{2} x_{5} \vee x_{1} x_{3} x_{4} \vee x_{2} x_{3} x_{4} \vee x_{3} x_{4} x_{5}$ type $(0,0,3,3,0,0)$ is not a maximal type MBF. To do this, in the conjunction $x_{1} x_{2}$ simultaneously replace $x_{1}$ with $x_{3}$ and $x_{2}$ with $x_{5}$, we obtain $g_{1}(5)=x_{1} x_{5} \vee x_{2} x_{5} \vee x_{3} x_{5} \vee x_{1} x_{3} x_{4} \vee x_{2} x_{3} x_{4} \vee x_{3} x_{4} x_{5}$. The resulting conjunction $x_{3} x_{5}$ absorbs the conjunction $x_{3} x_{4} x_{5}$, therefore, in $x_{3} x_{4} x_{5}$ we carry out the reverse change, i.e. simultaneously replace $x_{3}$ with $x_{1}$ and $x_{5}$ with $x_{2}$. we get $g_{2}(5)=x_{1} x_{5} \vee x_{2} x_{5} \vee x_{3} x_{5} \vee x_{1} x_{2} x_{4} \vee x_{1} x_{3} x_{4} \vee x_{2} x_{3} x_{4}$. The transition from $g(5)$ to $g_{2}(5)$ is called the second operation. Obviously $g_{1}(5), g_{2}(5)$ and $g(5)$, are of type $(0,0,3,3,0,0)$. But to $g_{2}(5)$, unlike $g(5)$, we can add the conjunction $x_{1} x_{2} x_{3}$. Get the MBF $g_{3}(5)=x_{1} x_{5} \vee x_{2} x_{5} \vee x_{3} x_{5} \vee x_{1} x_{2} x_{3} \vee x_{1} x_{2} x_{4} \vee$ $x_{1} x_{3} x_{4} \vee x_{2} x_{3} x_{4}$ type $(0,0,3,4,0,0)$. By the definition of the maximal type, neither $g_{2}(5)$ nor $g(5)$ are MBFs of maximal types.

Theorem 2. An MBF $f(n)$ of type $T$ with $\lambda=0$ of the second case is not an MBF of maximal type.

Proof. By the definition of the second case, there exists a component $i$ of type $T$ corresponding to component $i-1$ of type $T_{1}$ and containing 3 conjunctive clauses $A, B$ and $C$ that do not have common variables, and any variable $x_{k}$ belonging to two conjunctive clauses from $A, B$ and $C$ also belongs to some conjunctive clause $D$, which contains in 
component $i+1$ of type $T$. We apply the first operation to $B$ so that $A$ and $B$ have more common variables. We will repeat the first operation until the number of common variables in $A$ and $B$ becomes $i-1$. In the process of these operations, it may turn out that $B$ and $C$ become without common variables, i.e. the condition of Theorem 1 will be realized. In this case, the theorem is proved. Otherwise, we apply the first operation to $C$ several times, so that $C$ has separately with $A$ and $B i-1$ common variables, and together $A, B$ and $C$ have $i-2$ common variables. In this case, any variable from $C$ will belong to either $A$ or $B$. In total, these 3 conjunctive clauses will contain $\mathrm{i}+1$ variables. Denote the resulting conjunctive clauses $A, B$ ' and $C^{\prime}$. Type $T$ MBFs containing $A, B$, and $C^{\prime}$ are denoted by $f_{1}(n)$. For $A, B$ and $C$, maximum can be placed $C_{n}^{j}-3 C_{n-i}^{j-i}+3 C_{n-2 i+1}^{j-2 i+1}-$ $C_{n-3 i+3}^{j-3 i+3}$ conjunctive clauses belonging to component $j$ of type $T$. For $A, B^{\prime}$ and $C^{\prime}$, maximum can be placed $C_{n}^{j}-3$ $C_{n-i}^{j-i}+2 C_{n-i-1}^{j-i-1}$ conjunctive clauses belonging to component $j$ of type $T$. Denote a variable that belongs only to $A$ and $B^{\prime}$ through $x_{k}$, a variable belonging only to $A$ and $C^{\prime}$ through $x_{1}$, and a variable belonging only to $B^{\prime}$ and $C^{\prime}$ through $x_{m}$. As $x_{n}$, we take a variable that does not belong to any of the conjunctive clauses $A, B$ ', and $C^{\prime}$. We apply the second operation to $C^{\prime}$, replacing $x_{\mathrm{l}}$ with $x_{\mathrm{n}}$ and $x_{\mathrm{m}}$ with $x_{\mathrm{k}}$ at the same time. Then, in all conjunctive clauses absorbed by the resulting conjunctive clause $C^{\prime}$ ', we carry out the reverse change. Type $T$ MBFs containing $A, B$ ' and $C$ ', are denoted by $f_{2}(n)$. Obviously $f(n), f_{1}(n)$ and $f_{2}(n)$ are of one type $T$. For $A, B^{\prime}$ and $C^{\prime}$ ', maximum can be placed $C_{n}^{j}-3$ $C_{n-i}^{j-i}+3 C_{n-i-1}^{j-i-1}-C_{n-i-2}^{j-i-2}$ conjunctive clauses belonging to component $j$ of type $T$. The last expression is already for $j=$ $i+1$ is greater than the previous two. This means that type $T$ is not maximal. The theorem is proved.

Consequence. From MBF of maximal type, you can always take some variable $x_{k}$ out of the brackets, so that the types of the left and right sides will constitute a admissible pair of types. In other words, for any MBF of maximal type $\lambda>0$.

The distribution of MBFs by classes of isomorphic functions is conveniently presented in the form of diagrams, where we indicate the number of isomorphic MBFs in each class of rank $n-1$, the number of MBFs in class of rank $n-1$, which consists of classes of rank $n-1$. Further, in parentheses the index of separating variables in this class and in square brackets is the number of types for this scheme. Such schemes completely define the number of isomorphic and nonisomorphic MBFs in the type that belongs to this scheme, i.e. all types belonging to this scheme have the same number of isomorphic and nonisomorphic MBF.

Below are the schemes for rank 8. Tables of the dependence of the number of equivalence classes (the number of nonequivalent MBFs) and the number of all MBFs of maximal types on the power of the equivalence class (Table 1) and the number of equivalence classes obtained from 2 classes of $n-1$ rank (Table 2) are constructed.

Table 1. Kinds of classes MBF 8 rank

\begin{tabular}{|c|c|c|c|}
\hline $\begin{array}{l}\text { No. } \\
\text { p/p }\end{array}$ & $\begin{array}{c}\text { Cardinality of } \\
\text { class }\end{array}$ & $\begin{array}{c}\text { Number of } \\
\text { classes }\end{array}$ & $\begin{array}{c}\text { Number of } \\
\text { MBF }\end{array}$ \\
\hline 1 & 1 & 9 & 9 \\
\hline 2 & 8 & 21 & 168 \\
\hline 3 & 28 & 42 & 1,176 \\
\hline 4 & 56 & 72 & 4,032 \\
\hline 5 & 70 & 12 & 840 \\
\hline 6 & 168 & 186 & 31,248 \\
\hline 7 & 280 & 142 & 39,760 \\
\hline 8 & 336 & 179 & 60,144 \\
\hline 9 & 420 & 78 & 32,760 \\
\hline 10 & 560 & 56 & 31,360 \\
\hline 11 & 840 & 931 & 782,040 \\
\hline 12 & 1,120 & 418 & 468,160 \\
\hline 13 & 1,680 & 1,553 & $2,609,040$ \\
\hline 14 & 2,520 & 22 & 55,440 \\
\hline 15 & 3,360 & 5,202 & $17,478,720$ \\
\hline 16 & 5,040 & 2,622 & $13,214,880$ \\
\hline 17 & 6,720 & 4,389 & $29,494,080$ \\
\hline 18 & 10,080 & 15,388 & $155,111,040$ \\
\hline 19 & 20,160 & 23,601 & $475,796,160$ \\
\hline 20 & 40,320 & 11,013 & $444,044,160$ \\
\hline \multicolumn{2}{|r|}{ In total } & 65,936 & $1,139,255,217$ \\
\hline
\end{tabular}


Table 2. Kinds of MBF partitions of maximum types into classes

\begin{tabular}{|c|c|c|c|c|c|}
\hline No. $\mathrm{p} / \mathrm{p}$ & Cardinality of part & Num. of schemes & Num. of types & Num. of classes & Num. of MBF \\
\hline 1 & 1 & 32 & 1,574 & 1,574 & $3,528,009$ \\
\hline 2 & 2 & 15 & 359 & 718 & $2,102,296$ \\
\hline 3 & 3 & 19 & 605 & 1,815 & $8,028,720$ \\
\hline 4 & 4 & 13 & 298 & 1,192 & $6,909,616$ \\
\hline 5 & 5 & 8 & 312 & 1,560 & $10,758,720$ \\
\hline 6 & 6 & 5 & 314 & 1,884 & $13,432,272$ \\
\hline 7 & 7 & 5 & 97 & 679 & $4,803,792$ \\
\hline 8 & 8 & 8 & 338 & 2,704 & $25,737,600$ \\
\hline 9 & 9 & 2 & 78 & 702 & $7,902,720$ \\
\hline 10 & 10 & 3 & 46 & 460 & $7,728,432$ \\
\hline 11 & 11 & 3 & 164 & 1,804 & $19,356,960$ \\
\hline 12 & 12 & 4 & 90 & 1,080 & $13,592,880$ \\
\hline 13 & 13 & 6 & 128 & , 1,664 & $19,417,440$ \\
\hline 14 & 14 & 2 & 12 & 168 & $1,693,440$ \\
\hline 15 & 15 & 5 & 50 & 750 & $9,972,480$ \\
\hline 16 & 16 & 6 & 87 & 1,392 & $14,696,640$ \\
\hline 17 & 17 & 1 & 4 & 68 & 588,000 \\
\hline 18 & 18 & 1 & 48 & 864 & $16,934,400$ \\
\hline 19 & 19 & 2 & 42 & 798 & $9,172,800$ \\
\hline 20 & 21 & 1 & 8 & 168 & $2,116,800$ \\
\hline 21 & 22 & 3 & 12 & 264 & $3,575,040$ \\
\hline 22 & 23 & 1 & 32 & 736 & $11,289,600$ \\
\hline 23 & 25 & 4 & 113 & 2,825 & $38,455,200$ \\
\hline 24 & 28 & 1 & 16 & 448 & $5,644,800$ \\
\hline 25 & 29 & 1 & 8 & 232 & $3,104,640$ \\
\hline 26 & 30 & 4 & 88 & 2,640 & $44,452,800$ \\
\hline 27 & 31 & 1 & 4 & 124 & $1,683,440$ \\
\hline 28 & 33 & 1 & 32 & 1,056 & $18,616,000$ \\
\hline 29 & 34 & 2 & 20 & 680 & $8,937,600$ \\
\hline 30 & 36 & 2 & 36 & 1,296 & $25,119,360$ \\
\hline 31 & 37 & 2 & 26 & 962 & $18,345,600$ \\
\hline 32 & 39 & 1 & 50 & 1,950 & $35,280,000$ \\
\hline 33 & 40 & 1 & 16 & 640 & $9,408,000$ \\
\hline 34 & 41 & 1 & 8 & 328 & $5,174,400$ \\
\hline 35 & 44 & 1 & 4 & 176 & $2,822,500$ \\
\hline 36 & 46 & 2 & 16 & 736 & $16,934,400$ \\
\hline 37 & 50 & 1 & 4 & 200 & $3,136,000$ \\
\hline 38 & 51 & 1 & 40 & 2,040 & $42,336,000$ \\
\hline 39 & 54 & 2 & 36 & 1,944 & $41,865,600$ \\
\hline 40 & 56 & 1 & 4 & 224 & $5,080,320$ \\
\hline 41 & 58 & 2 & 8 & 464 & $11,289,600$ \\
\hline 42 & 59 & 1 & 24 & 1,416 & $25,401,600$ \\
\hline 43 & 67 & 1 & 25 & 1,675 & $35,280,000$ \\
\hline 44 & 72 & 1 & 12 & 864 & $16,934,400$ \\
\hline 45 & 75 & 1 & 8 & 600 & $12,700,800$ \\
\hline
\end{tabular}




\begin{tabular}{|c|c|c|c|c|c|}
\hline 46 & 81 & 1 & 10 & 810 & $21,168,000$ \\
\hline 47 & 86 & 1 & 4 & 344 & $8,467,200$ \\
\hline 48 & 87 & 2 & 64 & 5,568 & $127,008,000$ \\
\hline 49 & 93 & 1 & 4 & 372 & $8,467,200$ \\
\hline 50 & 108 & 1 & 12 & 1,296 & $28,224,000$ \\
\hline 51 & 114 & 1 & 16 & 1,920 & $21,168,000$ \\
\hline 52 & 120 & 1 & 8 & 968 & $50,803,200$ \\
\hline 53 & 121 & 1 & 4 & 572 & $14,401,600$ \\
\hline 54 & 143 & 1 & 10 & 616 & $42,112,000$ \\
\hline 55 & 148 & 1 & 8 & 1,512 & $16,934,400$ \\
\hline 56 & 154 & 1 & 1,480 & $42,336,000$ \\
\hline 57 & 189 & 1 & 8 & 968 & $50,803,200$ \\
\hline 58 & 207 & 1 & 1 & 378 & $28,224,000$ \\
\hline 59 & 242 & 1 & 5,461 & 65,936 & $12,700,800$ \\
\hline 60 & 378 & & & $1,139,255,217$ \\
\hline
\end{tabular}

Schemes of recursive construction of MBF 8 rank

1) $1.1 .1 \circ 1 \rightarrow 1(8)$ [9]; 2)1.2. $1 \circ 1 \rightarrow 8(1)[21]$;

3)1.3. $21 \circ 1 \rightarrow$ 28(6) [2]; 4)1.4. 7॰1 $\rightarrow$ 28(2) [30];

5) $1.5 .35 \circ 1 \rightarrow 56(5)[6]$; 6)1.6,,21॰1 $\rightarrow$ 56(3) [20];

7) $1.7 .7 \circ 1 \rightarrow 56(1)$ [40]; 8)1.8. 35॰1 $\rightarrow$ 70(4) [12];

9) 1.9. $42 \circ 1 \rightarrow$ 168(2) [20]; 10)1.10. 21॰1 $\rightarrow$ 168(1) [90]; 11)1.11. 140॰1 $\rightarrow$ 280(4) [2]; 12)1.12. 105॰1 $\rightarrow$ 280(3) [8]; 13)1.13. 35॰1 $\rightarrow$ 280(1) [80]; 14)1.14. 42॰1 $\rightarrow$ 336(1) [60]; 15)1.15. 210॰1 $\rightarrow$ 420(4) [2]; 16)1.16. 105॰1 $\rightarrow$ 420(2) [48];

17)1.17. $210 \circ 1 \rightarrow$ 560(3) [22]; 18)1.18. 140॰1 $\rightarrow$ 560(2) [22]; 19)1.19. 210॰1 $\rightarrow 840(2)$ [20]; 20)1.20. 105॰1 $\rightarrow 840(1)$ [196]; 21)1.21. $420 \circ 1 \rightarrow 1120(3)[4] ; 22) 1.22 .140 \circ 1 \rightarrow 1,120(1)$ [86];

23)1.23. $630 \circ 1 \rightarrow 1680(3)[10]$; 24)1.24. 420॰1 $\rightarrow 1680(2)[72] ;$ 25)1.25. 210॰1 $\rightarrow$ 1,680(1) [164];

26)1.26. $630 \circ 1 \rightarrow 2,520(2)[14] ;$

27)1.27. $840 \circ 1 \rightarrow 3,360(2)[10]$;

28)1.28. $420 \circ 1 \rightarrow 3,360(1)[254]$;

29)1.29. $1260 \circ 1 \rightarrow 5,040$ (2) [30];

30)1.30. 630॰1 $\rightarrow 5,040(1)[90]$;

31)1.31. 840॰1 $\rightarrow 6,720(1)[30]$;

32)1.32. $1,260 \circ 1 \rightarrow 10,080(1)$ [98];

33)2.1. $7 \circ 7 \rightarrow 28(2)+336(1)=364[10]$;

34) $2.2 .7 \circ 7 \rightarrow 56(1)+336(1)=392[5] ;$

35)2.3. $21 \circ 7 \rightarrow 168(2)+840(1)=1,008[8] ;$

36)2.4. $21 \circ 7 \rightarrow 336(1)+840(1)=1,176[44]$;

37)2.5. $35 \circ 7 \rightarrow 420(2)+1,120(1)=1,540[2]$;

38)2.6. $21 \circ 1+210 \circ 1 \rightarrow 168(1)+1,680(1)=1,848[18]$;

39) $2.7 .35 \circ 7 \rightarrow 840(1)+1,120(1)=1,960[54]$;

40)2.8. $42 \circ 1+210 \circ 1 \rightarrow 336(1)+1,680(1)=2,016[4] ;$

41)2.9. $105 \circ 1+840 \circ 1 \rightarrow 420(2)+3,360(2)=3,780$ [4]; 42)2.10. 105॰1 + 420॰1 $\rightarrow$ 840(1) + 3,360(1) = 4,200 [10];

43)2.11. $210 ॰ 1+420 \bullet 1 \rightarrow 1,680(1)+3,360(1)=5,040$ [70]; 44)2.12. 420॰1 + 420॰1 $\rightarrow 3,360(1)+3,360(1)=6,720$ [44];

45)2.13. $105 \circ 1+840 \circ 1 \rightarrow 840(1)+6,720(1)=7,560[14] ; 46) 2.14 .840 \circ 1+1260 \circ 1 \rightarrow 3,360(2)+5,040(2)=8,400$ [16];

47)2.15. $840 \circ 1+1260 \circ 1 \rightarrow 6720(1)+10080(1)=16800$ [56];

48)3.1. $42 \circ 7 \rightarrow 168(2)+336(1)+1,680(1)=2,184$ [2];

49)3.2. $42 \circ 7 \rightarrow 2 * 336(1)+1,680(1)=2,352$ [12];

50)3.3. $21 \circ 21 \rightarrow 56(3)+840(2)+1,680(1)=2,576[1]$;

51)3.4. $21 \circ 21 \rightarrow 168(1)+2 * 1,680(1)=3,528$ [44]; 
52)3.5. $105 \circ 7 \rightarrow 420(2)+1,680(1)+3,360(1)=5,460[16] ; 53) 3.6 .35 \circ 21 \rightarrow 840(1)+1,680(1)+3,360(1)=5,880[96]$; 54)3.7. $105 \circ 7 \rightarrow 840(1)+1,680(1)+3,360(1)=5,880[50] ; 55) 3.8 .140 \circ 7 \rightarrow 560(2)+2 * 3,360(1)=7,280[10]$ 56)3.9. $140 \circ 7 \rightarrow 1,120(1)+2 * 3,360(1)=7,840[20]$

57)3.10. 210॰7 $\rightarrow$ 1680(2) + 3360(1) + 5,040(1) = $10080[4]$

58)3.11. $2 * 210 \circ 1+840 \circ 1 \rightarrow 2 * 1680(1)+6720(1)=10080[8] ; 59) 3.12 .210 \circ 7 \rightarrow 2 * 3,360(1)+5,040(1)=11,760[46]$; 60)3.13. $105 \circ 1+630 \circ 1+840 \circ 1 \rightarrow 840(1)+5,040(1)+6,720(1)=12,600$ [72];

61)3.14. $2 * 420 \circ 1+1,260 \circ 1 \rightarrow 2 * 3,360(1)+10,080(1)=16,800[80]$;

62)3.15. $420 \circ 1+1,260 \circ 1+2,520 \circ 1 \rightarrow 1,680(2)+5,040(2)+10,080(2)=16,800[16]$

63)3.16. $210 \circ 1+840 \circ 1+1,260 \circ 1 \rightarrow 1,680(1)+6,720(1)+10,080(1)=18480[20]$;

64)3.17. $420 \circ 1+840 \circ 1+1260 \circ 1 \rightarrow 3,360(1)+6,720(1)+10,080(1)=20,160$ [34];

65)3.18. $3 * 1,260 \circ 1 \rightarrow 3 * 10,080(1)=30,240[18]$

66)3.19. $420 \circ 1+1,260 \circ 1+2,520 \circ 1 \rightarrow 3,360(1)+10,080(1)+20,160(1)=33,600$ [56];

67)4.1. $42 \circ 21 \rightarrow 336(1)+2 * 1680(1)+3360(1)=7056$ [26];

68)4.2. $35 \circ 35 \rightarrow 280(1)+1,120(1)+3,360(1)+5,040(1)=9,800[52]$;

69)4.3. $210 \circ 7 \rightarrow 840(2)+2 * 1680(1)+6720(1)=10920[2]$;

70)4.4. $210 \circ 7 \rightarrow 3 * 1,680(1)+6,720(1)=11,760[6] ;$

71)4.5. $42 \circ 35 \rightarrow 1,680(1)+3 * 3,360(1)=11,760[28]$;

72)4.6. $420 \circ 7 \rightarrow 1,680(2)+3,360(1)+6,720(1)+10,080(1)=21,840$ [8];

73)4.7. $2 * 140 \circ 1+2 * 1,260 \circ 1 \rightarrow 2 * 1,120(1)+2 * 10,080(1)=22,400$ [22];

74)4.8. $420 \circ 7 \rightarrow 2 * 3360(1)+6720(1)+10080(1)=23520$ [66];

75)4.9. $210 \circ 1+2 * 840 \circ 1+1,260 \circ 1 \rightarrow 1,680(1)+2 * 6,720(1)+10,080(1)=25,200[16]$;

76)4.10. $630 \circ 7 \rightarrow 2,520(2)+3 * 10,080(1)=32,760[4]$;

77)4.11. $2 * 840 \circ 1+2 * 1,260 \circ 1 \rightarrow 2 * 6,720(1)+2 * 10,080(1)=33,600$ [8];

78)4.12. $630 \circ 7 \rightarrow 5,040(1)+3 * 10,080(1)=32,760$ [34];

79)4.13. $2 * 1,260 \circ 1+2 * 2,520 \circ 1 \rightarrow 2 * 10,080(1)+2 * 20160(1)=60,480$ [26];

80)5.1. 105 $21 \rightarrow 840(2)+840(1)+1,680(2)+5,040(1)+6,720(1)=15,120$ [4];

81)5.2. $105 \circ 21 \rightarrow 840(1)+1,680(1)+3,360(1)+5,040(1)+6,720(1)=17,640$ [116];

82)5.3. $140 \circ 21 \rightarrow 2 * 1,680(2)+2 * 3,360(1)+10,080(1)=20,160$ [2];

83)5.4. $140 \circ 21 \rightarrow 4 * 3,360(1)+10,080(1)=23,520$ [52];

84)5.5. $840 \circ 7 \rightarrow 4 * 6,720(1)+20,160(1)=47,040[6]$;

85)5.6. $420 \circ 1+840 \circ 1+2 * 1,260 \circ 1+2,520 \circ 1 \rightarrow 3,360(1)+6,720(1)+2 * 10,080(1)+20,160(1)=50,400[108]$;

86)5.7. $1,260 \circ 7 \rightarrow 5,040(2)+2 * 10,080(1)+2 * 20,160(1)=65,520[6]$;

87)5.8. $1,260 \circ 7 \rightarrow 3 * 10,080(1)+2 * 20,160(1)=70,560[18]$;

88)6.1. $21 \circ 7+210 \circ 7 \rightarrow 336(1)+840(1)+3 * 1,680(1)+6,720(1)=12,936[2]$;

89)6.2. $105 \circ 35 \rightarrow 840(1)+2 * 3,360(1)+5,040(1)+6,720(1)+10,080(1)=29,400$ [132];

90)6.3. $210 \circ 21 \rightarrow 2 * 1,680(1)+5,040(1)+6,720(1)+2 * 10,080(1)=35,280$ [90];

91)6.4. $2 * 420 \circ 1+2 * 1,260 \circ 1+2 * 2,520 \circ 1 \rightarrow 2 * 3,360(1)+2 * 10,080(1)+2 * 20,160(1)=67,200$ [54];

92)6.5. $3 * 630 \circ 1+3 * 2,520 \circ 1 \rightarrow 3 * 5,040(1)+3 * 20,160(1)=75,600$ [36];

93)7.1. $42 \circ 42 \rightarrow 2 * 336(1)+4 * 1680(1)+6720(1)=14112[1]$

94)7.2. $105 \circ 7+420 \circ 7 \rightarrow 840(1)+1,680(1)+3 * 3,360(1)+6,720(1)+10,080(1)=29,400$ [2];

95)7.3. $210 \circ 21 \rightarrow 3 * 1,680(1)+3 * 6,720(1)+10,080(1)=35,280[16]$;

96)7.4. $140 \circ 35 \rightarrow 2 * 1,120(1)+2 * 3,360(1)+3 * 10,080(1)=39,200[60]$;

97)7.5. $6 * 1,260 \circ 1+5,040 \circ 1 \rightarrow 6 * 10,080(1)+40,320(1)=100,800[18]$;

98)8.1. $210 \circ 7+420 \circ 7 \rightarrow 3 * 1,680(1)+2 * 3,360(1)+2 * 6,720(1)+10,080(1)=35,280$ [4];

99)8.2. $105 \circ 42 \rightarrow 3 * 1,680(1)+2 * 3,360(1)+2 * 6,720(1)+10080(1)=35,280[14]$;

100)8.3. $420 \circ 7+420 \circ 7 \rightarrow 4 * 3,360(1)+2 * 6,720(1)+2 * 10,080(1)=47,040[4]$;

101)8.4. $140 \circ 42 \rightarrow 4 * 3,360(1)+2 * 6,720(1)+2 * 10,080(1)=47,040[6]$;

102)8.5. $105 \circ 7+840 \circ 7 \rightarrow 840(1)+1,680(1)+3,360(1)+4 * 6,720(1)+20,160(1)=52,920[4]$;

103)8.6. $210 \circ 35 \rightarrow 1,680(1)+2 * 3,360(1)+2 * 5,040(1)+2 * 10,080(1)+20,160(1)=58,800[116]$;

104)8.7. $420 \circ 21 \rightarrow 2 * 3,360(1)+2 * 6,720(1)+3 * 10,080(1)+20,160(1)=70,560$ [138];

105)8.8. $3 * 1,260 \circ 1+4 * 2,520 \circ 1+5,040 \circ 1 \rightarrow 3 * 10,080(1)+4 * 20,160(1)+40,320(1)=151,200[52]$;

106)9.1. $210 \circ 42 \rightarrow 2 * 3,360(1)+2 * 6720(1)+5 * 10,080(1)=70,560[10]$;

107)9.2. $630 \circ 21 \rightarrow 3 * 5,040(1)+3 * 10,080(1)+3 * 20,160(1)=105,840$ [68];

108)10.1. $21 \cdot 21+210 \cdot 21 \rightarrow 168(1)+5^{*} 1,680(1)+3 * 6,720(1)+10,080(1)=38,808[4]$;

109)10.2. $840 \circ 7+1,260 \circ 7 \rightarrow 4 * 6,720(1)+3 * 10,080(1)+3 * 20,160(1)=117,600[16]$;

110)10.3. $4 * 1,260 \circ 1+4 * 2,520 \circ 1+2 * 5,040 \circ 1 \rightarrow 4 * 10,080(1)+4 * 20,160(1)+2 * 40,320(1)=201,600[26]$; 
111) 11.1. $21 \circ 35+210 \circ 35 \rightarrow 840(1)+2 * 1,680(1)+3,360(1)+4 * 6,720(1)+3 * 10,080(1)=64,680$ [4];

112)11.2. $420 \circ 35 \rightarrow 3 * 3,360(1)+6,720(1)+4 * 10,080(1)+3 * 20,160(1)=117,600$ [148];

113)11.3. $840 \circ 21 \rightarrow 6 * 6720(1)+5 * 20160(1)=141120[12]$;

114)12.1. $105 \circ 105 \rightarrow 420(2)+1,680(1)+2,520(2)+3360(2)+3360(1)+3 * 6720(1)+3 * 10080(1)+20160(1)=81900$

[4];

115)12.2. $105 \circ 105 \rightarrow 840(1)+1,680(1)+3,360(1)+5040(1)+4 * 6,720(1)+3 * 10,080(1)+20,160(1)=88,200[32] ;$

116) $12.3 .105 \circ 7+630 \circ 7+840 \circ 7 \rightarrow 840(1)+1680(1)+3360(1)+5040(1)+4 * 6720(1)+3 * 10080(1)+20160(1)=88200$ [8];

117)12.4. $1,260 \circ 21 \rightarrow 5 * 10,080(1)+6 * 20,160(1)+40320(1)=211,680[46]$

[4];

118) $13.1 .105 \cdot 21+420 \circ 21 \rightarrow 840(1)+1680(1)+3 * 3360(1)+5040(1)+3 * 6720(1)+3 * 10080(1)+20160(1)=88200$

119)13.2. $140 \circ 105 \rightarrow 2 * 1,680(2)+2 * 3,360(1)+5,040(2)+2 * 6,720(1)+4 * 10,080(1)+2 * 20,160(1)=109,200[4]$;

120)13.3. $140 \circ 105 \rightarrow 4 * 3,360(1)+2 * 6,720(1)+5 * 10,080(1)+2 * 20,160(1)=117,600$ [32];

121)13.4. $2 * 420 \circ 7+1,260 \circ 7 \rightarrow 4 * 3,360(1)+2 * 6,720(1)+5 * 10,080(1)+2 * 20,160(1)=117,600[8]$;

122) $13.5 .210 \circ 7+840 \circ 7+1,260 \circ 7 \rightarrow 2 * 3,360(1)+5,040(1)+4 * 6,720(1)+3 * 10,080(1)+3 * 20,160(1)=129,360[4]$;

123)13.6. $630 \circ 35 \rightarrow 3 * 5,040(1)+6 * 10,080(1)+3 * 20,160(1)+40,320(1)=176,400[76]$;

124)14.1. $420 \circ 7+840 \circ 7+1260 \circ 7 \rightarrow 2 * 3360(1)+5 * 6720(1)+4 * 10,080(1)+3 * 10,080(1)+20,160(1)=141,120[2]$;

125)14.2. $420 \circ 42 \rightarrow 2 * 3,360(1)+5 * 6,720(1)+4 * 10,080(1)+3 * 20,160(1)=141,120[10]$;

126)15.1. $210 \circ 21+420 \circ 21 \rightarrow 3 * 1,680(1)+2 * 3,360(1)+5 * 6,720(1)+4 * 10,080(1)+20,160(1)=105,840[12]$;

127) $15.2 .3 * 1260 \circ 7 \rightarrow 9 * 10080(1)+6 * 20160(1)=211680[2]$;

128)15.3. $630 \circ 42 \rightarrow 9 * 10,080(1)+6 * 20160(1)=211680[8]$;

129) $15.4 .420 \circ 7+1,260 \circ 7+2520 \circ 7 \rightarrow 2 * 3360(1)+6720(1)+4 * 10080(1)+7 * 20160(1)+40320(1)=235200[16]$;

130)15.5. $840 \circ 35 \rightarrow 5 * 6720(1)+10 * 20160(1)=235200[12]$;

131)16.1. $420 \circ 21+420 \circ 21 \rightarrow 4 * 3,360(1)+4 * 6,720(1)+6 * 10,080(1)+2 * 20,160(1)=141,120[8] ;$

132)16.2. $140 \circ 140 \rightarrow 2 * 560(2)+4 * 3,360(1)+2 * 5,040(2)+4 * 10,080(1)+4 * 20,160(1)=145,600[1]$;

133)16.3. $2 * 140 \circ 7+2 * 1,260 \circ 7 \rightarrow 2 * 1,120(1)+4 * 3,360(1)+6 * 10,080(1)+4 * 20,160(1)=156,800[2]$;

134)16.4. $140 \circ 140 \rightarrow 2 * 1,120(1)+4 * 3,360(1)+6 * 10,080(1)+4 * 20,160(1)=156,800[8]$;

135)16.5. $105 \circ 21+840 \circ 21 \rightarrow 840(1)+1,680(1)+3,360(1)+5,040(1)+7 * 6,720(1)+5 * 20,160(1)=158,760[8]$;

136)16.6. $210 \circ 105 \rightarrow 2 * 3,360(1)+3 * 5,040(1)+2 * 6,720(1)+4 * 10,080(1)+5 * 20,160(1)=176,400[60]$;

137)17.1. $105 \circ 35+420 \circ 35 \rightarrow 840(1)+5 * 3360(1)+5,040(1)+2 * 6,720(1)+5 * 10,080(1)+3 * 20,160(1)=147,000[4] ;$

138)18.1. $1,260 \circ 35 \rightarrow 7 * 10,080(1)+8 * 20,160(1)+3 * 40,320(1)=352,800[48]$;

139)19.1. $210 \circ 35+420 \circ 35 \rightarrow 1,680(1)+3 * 3,360(1)+5 * 6720(1)+7 * 10,080(1)+3 * 20,160(1)=176,400[12]$;

140)19.2. $210 \circ 140 \rightarrow 4 * 3,360(1)+10 * 10,080(1)+4 * 20160(1)+40,320(1)=235,200[30]$;

141)21.1(20.1) $105 \circ 35+840 \circ 35 \rightarrow 840(1)+2 * 3360(1)+5040(1)+6 * 6720(1)+10080(1)+10 * 20160(1)=264600$ [8];

142)22.1(21.1) $420 \circ 35+420 \circ 35 \rightarrow 6 * 3,360(1)+2 * 6,720(1)+8 * 10,080(1)+6 * 20,160(1)=235,200$ [8];

143)22.2(21.2) $2 * 1,260 \circ 7+2 * 2,520 \circ 7 \rightarrow 6 * 10,080(1)+14 * 20,160(1)+2 * 40,320(1)=423,360[2]$;

144)22.3(21.3) $1,260 \circ 42 \rightarrow 6 * 10,080(1)+14 * 20,160(1)+2 * 40,320(1)=423,360$ [2];

145)23.1(22.1) $840 \circ 21+1,260 \circ 21 \rightarrow 6 * 6,720(1)+5 * 10080(1)+11 * 20,160(1)+40,320(1)=352,800$ [32];

146)25.1(23.1) $105 \circ 21+630 \circ 21+840 \circ 21 \rightarrow 840(1)+1680(1)+3,360(1)+4 * 5,040(1)+7 * 6,720(1)+3 * 10,080(1)+$ $8 * 20,160(1)=264,600[16]$;

147)25.2(23.2) $420 \circ 7+840 \circ 7+2 * 1,260 \circ 7+2,520 \circ 7 \rightarrow 2 * 3,360(1)+5 * 6,720(1)+7 * 10,080(1)+10 * 20,160(1)+$ $40,320(1)=352,800[12]$;

148)25.3(23.3) $420 \circ 105 \rightarrow 2 * 3,360(1)+5 * 6,720(1)+7 * 10080(1)+10 * 20,160(1)+40,320(1)=352,800[60]$;

149) $25.4(23.4) 210 \circ 210 \rightarrow 2 * 1680(1)+4 * 5040(1)+6720(1)+8 * 10080(1)+8 * 20160(1)+2 * 40320(1)=352800$ [25]; 150)28.1(24.1) $420 \circ 21+420 \circ 21+1,260 \circ 21 \rightarrow 4 * 3360(1)+4 * 6720(1)+11 * 10080(1)+8 * 20160(1)+40320(1)=352800$ [16];

151)29.1(25.1) $210 \circ 21+840 \circ 21+1,260 \circ 21 \rightarrow 2 * 1,680(1)+5,040(1)+7 * 6,720(1)+7 * 10,080(1)+11 * 20,160(1)+$ $40,320(1)=388,080[8]$;

152)30.1(26.1) $2 *(420 \circ 7+1,260 \circ 7+2,520 \circ 7) \rightarrow 4 * 3,360(1)+2 * 6,720(1)+8 * 10,080(1)+14 * 20,160(1)+$ $2 * 40,320(1)=470,400[6]$

153)30.2(26.2) $420 \circ 140 \rightarrow 4 * 3,360(1)+2 * 6,720(1)+8 * 10080(1)+14 * 20,160(1)+2 * 40,320(1)=470,400$ [30];

154)30.3(26.3) $3 * 630 \circ 7+3 * 2520 \circ 7 \rightarrow 3 * 5040(1)+9 * 10080(1)+15 * 20,160(1)+3 * 40,320(1)=529,200[4]$;

155)30.4(26.4) 630॰105 $\rightarrow 3 * 5,040(1)+9 * 10,080(1)+15 * 20,160(1)+3 * 40,320(1)=529,200$ [48];

156)31.1(27.1) $420 \circ 21+840 \circ 21+1,260 \circ 21 \rightarrow 2 * 3,360(1)+8 * 6,720(1)+8 * 10,080(1)+12 * 20,160(1)+40,320(1)=$ 423,360 [4];

157)33.1(28.1) $840 \circ 35+1,260 \circ 35 \rightarrow 5 * 6,720(1)+7 * 10080(1)+18 * 20,160(1)+3 * 40,320(1)=588,000$ [32];

158)34.1(29.1) $105 \circ 35+630 \circ 35+840 \circ 35 \rightarrow 840(1)+2 * 3,360(1)+4 * 5,040(1)+6 * 6,720(1)+7 * 10,080(1)+$ 
$13 * 20,160(1)++40,320(1)=441,000[16] ;$

159)34.2(29.2) $2 * 140 \circ 21+2 * 1,260 \circ 21 \rightarrow 8 * 3,360(1)+12 * 10,080(1)+12 * 20,160(1)+2 * 40,320(1)=470,400$ [4];

160)36.1(30.1) $3 * 1,260 \circ 21 \rightarrow 15 * 10,080(1)+18 * 20,160(1)+3 * 40,320(1)=635,040$ [4];

161)36.2(30.2) $420 \circ 21+1,260 \circ 21+2,520 \circ 21 \rightarrow 2 * 3,360(1)+2 * 6,720(1)+8 * 10,080(1)+18 * 20,160(1)+6 * 40,320(1)$

$=705,600[36]$;

162)37.1(31.1) $6 * 1,260 \circ 7+5,040 \circ 7 \rightarrow 18 * 10,080(1)+12 * 20,160(1)+7 * 40,320(1)=705,600[2] ;$

163)37.2(31.2) $630 \circ 140 \rightarrow 18 * 10,080(1)+12 * 20,160(1)+7 * 40,320(1)=705,600[24]$;

164)39.1(32.1) $420 \circ 210 \rightarrow 2 * 3,360(1)+2 * 6,720(1)+12 * 10080(1)+18 * 20,160(1)+5 * 40,320(1)=705,600$ [50];

165) $40.1(33.1) 2 * 420 \circ 35+1,260 \circ 35 \rightarrow 6 * 3,360(1)+2 * 6,720(1)+15 * 10,080(1)+14 * 20,160(1)+3 * 40,320(1)=$ 588,000 [16];

166) $41.1(34.1) 210 \circ 35+840 \circ 35+1,260 \bullet 35 \rightarrow 1,680(1)+2 * 3,360(1)+2 * 5,040(1)+5 * 6,720(1)+9 * 10,080(1)+$ $19 * 20,160(1)++3 * 40,320(1)=646,800[8]$

167)44.1(35.1) $420 \circ 35+840 \circ 35+1,260 \circ 35 \rightarrow 3 * 3,360(1)+6 * 6,720(1)+11 * 10,080(1)+21 * 20,160(1)+3 * 40,320(1)$ $=705,600[4]$;

168)46.1(36.1) $3 * 1,260 \circ 7+4 * 2,520 \circ 7+5,040 \circ 7 \rightarrow 9 * 10080(1)+26 * 20,160(1)+11 * 40,320(1)=1,058,400[4]$;

169)46.2(36.2) $1,260 \circ 105 \rightarrow 9 * 10,080(1)+26 * 20,160(1)+11 * 40,320(1)=1,058,400[12]$;

170)50.1(37.1) $2 * 140 \circ 35+2 * 1,260 \circ 35 \rightarrow 4 * 1,120(1)+4 * 3,360(1)+20 * 10,080(1)+16 * 20,160(1)+6 * 40,320(1)=$ 784,000 [4];

171)51.1(38.1) $630 \circ 210 \rightarrow 6 * 5,040(1)+12 * 10,080(1)+21 * 20,160(1)+12 * 40,320(1)=1,058,400$ [40];

172)54.1(39.1) $3 * 1,260 \circ 35 \rightarrow 21 * 10,080(1)+24 * 20,160(1)+9 * 40,320(1)=1,058,400$ [4];

173) $54.2(39.2) 420 \circ 35+1,260 \circ 35+2,520 \circ 35 \rightarrow 3 * 3,360(1)+6,720(1)+11 * 10,080(1)+26 * 20,160(1)+13 * 40,320(1)$ $=1,176,000$ [32];

174)56.1(40.1) $2 * 1,260 \circ 21+2 * 2,520 \circ 21 \rightarrow 10 * 10,080(1)+34 * 20,160(1)+12 * 40,320(1)=1,270,080[4]$;

175)58.1(41.1) $4 * 1,260 \circ 7+4 * 2,520 \circ 7+2 * 5,040 \circ 7 \rightarrow 12 * 10080(1)+28 * 20160(1)+18 * 40320(1)=1,411,200[2]$;

176)58.2(41.2) $1,260 \circ 140 \rightarrow 12 * 10,080(1)+28 * 20,160(1)+18 * 40,320(1)=1,411,200[6]$;

177)59.1(42.1) $420 \circ 21+840 \circ 21+2 * 1,260 \circ 21+2,520 \circ 21 \rightarrow 2 * 3,360(1)+8 * 6,720(1)+13 * 10,080(1)+29 * 20,160(1)$

$+7 * 40,320(1)=1,058,400[24]$

178)67.1(43.1) $420 \circ 420 \rightarrow 2 * 3,360(1)+5 * 6,720(1)+10 * 10080(1)+37 * 20160(1)+13 * 40320(1)=1411200$ [25];

179)72.1(44.1) $2 * 420 \circ 21+2 * 1,260 \circ 21+2 * 2520 \circ 21 \rightarrow 4 * 3360(1)+4 * 6,720(1)+16 * 10,080(1)+36 * 20160(1)+$ $12 * 40,320(1)=1,411,200[12]$;

180)75.1(45.1) $3 * 630 \circ 21+3 * 2,520 \circ 21 \rightarrow 9 * 5,040(1)+9 * 10080(1)+42 * 20,160(1)+15 * 40,320(1)=1,587,600[8]$;

181)81.1(46.1) $1,260 \circ 210 \rightarrow 14 * 10,080(1)+36 * 20,160(1)+31 * 40,320(1)=2,116,800$ [10];

182)86.1(47.1) $2 * 1,260 \circ 35+2 * 2,520 \circ 35 \rightarrow 14 * 10,080(1)+46 * 20,160(1)+26 * 40,320(1)=2,116,800[4]$;

183)87.1(48.1) $420 \circ 35+840 \circ 35+2 * 1,260 \circ 35+2,520 \circ 35 \rightarrow 3 * 3,360(1)+6 * 6,720(1)+2 * 9 * 10,080(1)+44 * 20,160(1)$

$++16 * 40,320(1)=1,764,000[24]$.

184)87.2(48.2,630 $420 \rightarrow 18 * 10,080(1)+42 * 20,160(1)+27 * 40,320(1)=2,116,800[40] ;$

185)93.1(49.1) $6 * 1,260 \circ 21+5,040 \circ 21 \rightarrow 30 * 10,080(1)+36 * 20,160(1)+27 * 40,320(1)=2,116,800$ [4];

186)108.1(50.1) $2 * 420 \circ 35+2 * 1,260 \circ 35+2 * 2,520 \circ 35 \rightarrow 6 * 3,360(1)+2 * 6,720(1)+22 * 10,080(1)+52 * 20,160(1)+$ $26 * 40,320(1)=2,352,000[12]$;

187)114.1(51.1) $3 * 630 \circ 35+3 * 2,520 \circ 35 \rightarrow 9 * 5,040(1)+18 * 10080(1)+54 * 20160(1)+33 * 40320(1)=2646000$ [8];

188)120.1(52.1) $630 \circ 630 \rightarrow 6 * 5,040(1)+18 * 10,080(1)+45 * 20,160(1)+51 * 40,320(1)=3,175,200[16]$;

189)121.1(53.1) $3 * 1,260 \circ 21+4 * 2,520 \circ 21+5040 \circ 21 \rightarrow 15 * 10,080(1)+62 * 20,160(1)+44 * 40,320(1)=3175,200$ [8];

190)143.1(54.1) $6 * 1,260 \circ 35+5,040 \circ 35 \rightarrow 42 * 10,080(1)+48 * 20,160(1)+53 * 40,320(1)=3,528,000$ [4];

191)148.1(55.1) $1,260 \circ 420 \rightarrow 12 * 10,080(1)+68 * 20,160(1)+68 * 40,320(1)=4,233,600[10] ;$

192)154.1(56.1) $4 * 1,260 \circ 21+4 * 2520 \circ 21+2 * 5040 \circ 21 \rightarrow 20 * 10080(1)+68 * 20160(1)+66 * 40320(1)=4,233,600$

[4];

193)189.1(57.1) $3 * 1,260 \bullet 35+4 * 2,520 \bullet 35+5,040 \rightarrow 21 * 10080(1)+84 * 20160(1)+84 * 40320(1)=5,292,000$ [8];

194)207.1(58.1) $1,260 \circ 630 \rightarrow 18 * 10,080(1)+72 * 20,160(1)+117 * 40,320(1)=6,350,400$ [8];

195)242.1(59.1) $4 * 1,260 \bullet 35+4 * 2520 \bullet 35+2 * 5040 \bullet 35 \rightarrow 28 * 10080(1)+92 * 20160(1)+122 * 40320(1)=7056000$

[4];

196)378.1(60.1) $1260 \circ 1260 \rightarrow 12 * 10080(1)+108 * 20160(1)+258 * 40,320(1)=12,700,800[1]$. 
In [9], formulas were found for finding the number of MBFs $n$th rank and classes of functions $n$th rank obtained from two classes of $n-1$ rank:

Theorem 3. The number of MBF $n$-th rank obtained from pairs $\left(f_{i}, g_{j}\right)$ of MBF $n-1$ rank of classes $L$ and $R$ is computed by the formula $K=\sum_{i=1}^{l} \frac{k_{i} r n}{\lambda_{i}}$

Theorem 4. The total number classes of functions nth rank obtained from the two classes $n-1$ of the rank $L$ and $R$ are $l=\sum_{j=1}^{v} \frac{t_{j} w_{j}}{a}$

Here we use the notation from [9], $k_{i}=\frac{\left|S t_{1}\right|}{\left|S t_{1} \cap S t_{i}^{\prime}\right|}$, $a=\frac{(n-1) !}{r}=\left|S t_{1}\right|, \quad r$ - the number of isomorphic left MBFs, $w_{1}, \ldots, w_{v}$ - the intersection cardinality $S t_{1} \cap S t_{i}^{\prime}, t_{i}$ - the number of functions corresponding to the intersection $w_{i}$.

Consider an example for rank 8.

EXAMPLE 4. Take the left MBF of the 7th rank, having the type $(0,3,6,0,0,0,0,0,0)$

$f_{1}(7)=x_{1} \vee x_{2} \vee x_{3} \vee x_{4} x_{5} \vee x_{4} x_{6} \vee x_{4} x_{7} \vee x_{5} x_{6} \vee x_{5} x_{7} \vee x_{6} x_{7}$ . From it 35 isomorphic MBFs are obtained. The stabilizer $S t_{1}$ of this function consists of 144 permutations. Take the right MBF of the 7th rank, having type (0,0,0,0,0,6,3,0):

$$
\begin{aligned}
g_{1}(5)= & x_{1} x_{2} x_{3} x_{4} x_{5} \vee x_{1} x_{2} x_{3} x_{4} x_{6} \vee x_{1} x_{2} x_{3} x_{4} x_{7} \vee x_{1} x_{2} x_{3} x_{5} x_{6} \vee \\
& \vee x_{1} x_{2} x_{3} x_{5} x_{7} \vee x_{1} x_{2} x_{3} x_{6} x_{7} \vee x_{1} x_{2} x_{4} x_{5} x_{6} x_{7} \vee \\
& \vee x_{1} x_{3} x_{4} x_{5} x_{6} x_{7} \vee x_{2} x_{3} x_{4} x_{5} x_{6} x_{7}
\end{aligned}
$$

It also produces 35 isomorphic MBFs. The stabilizer of this function also consists of 144 permutations.

There are only 4 different cardinality intersections $S t_{1}$ with all 35 stabilizers $S t_{1}^{\prime}, \ldots, S t^{\prime}{ }_{35}$ of the right functions. The cardinality of these intersections $S t_{1}$ with $S t_{1}{ }_{1}, \ldots, S t^{\prime}{ }_{35}$ are: $8, \quad 12, \quad 36, \quad 144$. That is $w_{1}=8, w_{2}=12, w_{3}=36, w_{4}=144$. We calculate the number of right functions corresponding to the intersections $8, \quad 12, \quad 36, \quad 144$ will be $t_{1}=18, t_{2}=12, t_{3}=4, t_{4}=1$. Then total of such classes of functions of the eighth rank (Theorem 4) $l=\sum_{j=1}^{v} \frac{t_{j} w_{j}}{a}=\frac{18 \cdot 8}{144}+\frac{12 \cdot 12}{144}+\frac{4 \cdot 36}{144}+\frac{1 \cdot 144}{144}=4$. In all 4 classes, the index of separating variables $\lambda=1$. Now we calculate the number of MBFs in each class and the total number of MBFs of the eighth rank having type $(0,0,3,6,0,6,3,0,0)$. (Theorem 3)

$$
\begin{aligned}
K & =\sum_{i=1}^{l} \frac{k_{i} r n}{\lambda_{i}}=\frac{18 \cdot 35 \cdot 8}{1}+\frac{12 \cdot 35 \cdot 8}{1}+\frac{4 \cdot 35 \cdot 8}{1}+\frac{1 \cdot 35 \cdot 8}{1}= \\
& =5040+3360+1120+280=9800,
\end{aligned}
$$

here $r=35$ is the number of isomorphic left MBFs.

The scheme corresponding to this example is 4.2 under number 68 .

68) $4.2 .35 \circ 35 \rightarrow 280(1)+1$ 120(1)+3 360(1)+5 040(1) = $=9800$ [52]

This shows that the same scheme, i.e. 35 left and 35 right MBFs have a total of 52 types including 4 isomorphic classes each, which consist of 280, 1120, 3360, 5040 MBFs. In total, this scheme is $52 \cdot 4=208$ isomorphic classes.

It can be noted that the class, which includes $280 \mathrm{MBFs}$, consists of disjunctively self-complementary functions, i.e. MBFs in which for each conjunctive clause there is another conjunctive clause consisting of variables not included in the first conjunctive clauses.

\section{Conclusions}

This paper is the first to introduce a method for constructing and analyzing MBFs of maximal eighth rank types. The schemes of such MBFs are presented. Using the schemes allows split 1,139,255,217 MBFs of maximal types of rank 8 into 196 schemes. In fact, in the program contains only nonequivalent MBFs, and the sizes of equivalent classes are calculated by the formula [9]. After some refinement of the program, it is possible to conduct a similar analysis of the MBF of the ninth rank. For non-maximal types, such an analysis is much more difficult to carry out and this requires further research.

\section{REFERENCES}

[1] Dedekind R. Uber Zerlegungen von Zahlen durch ihre gr " " ossten gemainsamen Teilor // Festschrift Hoch. Braunschweig u. ges. Werke. II. - 1897. — S. 103-148.

[2] N. J. A. Sloane, The online encyclopedia of integer sequences, http://oeis.org, 2011.

[3] K. Engel, Sperner theory, Cambridge University Press, 1997.

[4] T. Stephen, T. Yusun. Counting inequivalent monotone boolean functions. arXiv:1209.4623v1 [cs.DS] 20 Sep 2012

[5] Wiedemann, Doug (1991) A computation of the eighth Dedekind number. Order Vol. 8, no. 1, pp. 5-6.

[6] Ткаченко В.Г. Классификация монотонных булевых функций при синтезе цифровых схем / В.Г. Ткаченко // Наукові праці ОНАЗ ім. О.С. Попова. - 2008. - № 1. - С. $35-43$.

[7] Ткаченко В.Г. Перечисление типов монотонных булевых функций при синтезе цифровых схем / В.Г. Ткаченко // Наукові праці ОНАЗ ім. О.С. Попова. - 2008. - № 2. - C. $54-69$.

[8] Tkachenco V.G., Sinyavsky O. V. (2017). Algebraic Objects of MBFs and Recursive Computation of the Dedekind 
Number, Computer Science and Information Technology Vol. 5(4), pp. 140 - 147 DOI: 10.13189/csit.2017.050404

[9] Tkachenco V. G., Sinyavsky O. V. (2019). Analysis and Recurrent Computation of MBF of the Maximum Types. Computer Science and Information Technology, Vol. 7, №3. - pp. 72- 81. DOI: 10.13189/csit.2019.070303

[10] Tkachenco V. G., Sinyavsky O. V. (2018). Maximum MBF Types. Computer Science and Information Technology, Vol. 6, №4. - pp. 47- 53. DOI: 10.13189/csit.2018.060401. 